Original

\title{
Incontinencia de orina durante la actividad sexual coital. Sintomas asociados y gravedad de la incontinencia
}

\author{
Montserrat Espuña-Pons, Montserrat Puig-Clota \\ Institut Clínic de Ginecología, Obstetricia i Neonatología. Hospital Clinic I Provincial. Universidad de Barcelona. \\ España
}

\section{Resumen}

Introducción: En el presente estudio se analizó en una muestra de mujeres sexualmente activas que consultaban por incontinencia urinaria (IU), la relación entre la IU en el coito y los síntomas de IU de esfuerzo y de urgencia y el grado de afectación por la IU expresados por la mujer mediante el cuestionario ICIQ-UI SF.

Material y métodos: Estudio epidemiológico, observacional, transversal y multicéntrico de 1.735 mujeres con diagnóstico de IU. Las pacientes que no tenían relación sexual de pareja fueron excluidas siendo analizadas 1.292 mujeres, que cumplimentaron el cuestionario ICIQ-UI SF y el King's Health Questionnaire (KHQ).

Resultados: De acuerdo al KHQ, la prevalencia de IU en el coito fue de 29,4\%. Las mujeres con IU en el coito en comparación a las que no la padecían presentaron similar edad media e índice de masa corporal, con mayor frecuencia síntomas de IUE $(37,6 \%$ vs $27,7 \%)$ y con menor frecuencia síntomas de IUU (16,6\% vs 27,4\%) (p<0,001). El 36,1\% de las mujeres con IUE referían IU en el coito, porcentaje que resultó inferior en las mujeres con IUU (20,1\%). La puntuación total del ICIQ-UI SF fue superior en mujeres con IU en el coito, 14,1 frente a 12,1 ( $<<0,001)$, diferencia debida fundamentalmente a la dimensión "afectación" más que a "frecuencia" y "cantidad".

Discusión: La IU en el coito parece asociarse a la presencia de síntomas de IUE y a una mayor gravedad de la IU medidos por el cuestionario ICIQ-UI SF. Probablemente esta asociación se deba a que el ICIQ-UI SF incluye en su puntuación total la afectación causada por la IU y la IU en el coito tiene un importante efecto en la calidad de vida de las mujeres sexualmente activas.

Palabras clave. Incontinencia Urinaria. Coito. Mujer. Cuestionarios. Calidad de vida.

\section{Coital Urinary incontinence. Associated symptoms and severity of incontinence.}

\section{Abstract}

Introduction: The objective of present study was to analyze the relationship between coital urinary incontinence (UI) and the symptoms of Stress UI (SUI), Urge UI (UUI) and the extent to which they impair the patient's life, measured through the ICIQ-UI SF questionnaire in a sample of sexually active women with UI symptoms.

Methods: Epidemiologic, observational, cross-sectional and multicentric study of 1,735 women with diagnostic of urinary incontinence (UI). Patients who were not sexually active were excluded, remaining 1,292 patients for the analysis. All women filled in the ICIQ-UI SF questionnaire and the King’s Health Questionnaire (KHQ).

Results: According to KHQ, the prevalence of coital incontinence was $29.4 \%$. Women with coital incontinence compared to those who did not suffer from it, had similar mean age and body mass index , were more likely to have symptoms of SUI (37.6\% vs. $27.7 \%)$ and less likely to have UUI (16.6\% vs. $27.4 \%)(\mathrm{p}<0.001) .36 .1 \%$ of women with SUI had coital UI; this percentage was lower for women with UUI (20.1\%). ICIQ-UI global score was higher in women with coital incontinence, 14.1 vs. 12.1 ( $<<0.001)$, difference that was mainly due to the dimension "affectation" rather than "frequency" and "amount" dimension scores.

Discussion: Coital IU seem to be associated to SUI and to a higher severity of the urinary incontinence measured by the ICIQ-UI SF. Probably this association is due to the fact that ICIQ-UI-SF includes in its score the impact of patients' UI symptoms on their life and coital incontinence has an important effect on the quality of life of sexually active women.

Keywords: Urinary incontinence. Coitus. Women- Questionnaires. Quality of life.

$L^{2}$ a incontinencia urinaria (IU) en el coito es un problema que se presenta en una tercera parte de las mujeres sexualmente activas con síntomas urinarios $^{1}$ y que causa un gran impacto en la calidad de vida ${ }^{2}$. De hecho la IU en el coito se asocia a peor calidad de vida independientemente de la edad, el IMC y otros sintomas urinarios ${ }^{1}$. La importancia de este síntoma urinario se ha puesto de relieve en el estudio de $\mathrm{Oh}$ et al recientemente publicado ${ }^{3}$ en el que se encontró que las mujeres con IU en el coito tenían más frecuentemente otros sintomas urinarios (urgencia, IU de urgencia, dolor vesical, IU de 
esfuerzo, nocturia y disfunción de vaciado) que las que no la tenían; y se comprobó también el gran impacto en la calidad de vida que causa la presencia de este síntoma.

Según algunos autores ${ }^{4}$ no existen diferencias en la función sexual de las mujeres con diferentes diagnósticos de IU (IU de Esfuerzo, Hiperactividad del Detrusor o IU Mixta). Sin embargo los resultados de otro estudio ${ }^{5}$ sugieren que las mujeres con IU de Esfuerzo (IUE) experimentan más frecuentemente dolor e IU en el coito que las mujeres con IU de Urgencia (IUU). Recientemente se ha empezado a diferenciar entre IU con el orgasmo e IU con la penetración ${ }^{6}$; parece que la IU con el orgasmo se asocia al hallazgo urodinámico de hiperactividad del detrusor con una frecuencia significativamente mayor $(69,4 \%)$ que la IU con la penetración $(28,9 \%)$.

El presente estudio se realizó con el objetivo de analizar la relación entre el síntoma IU en el coito y los síntomas de IU de esfuerzo y de urgencia y el grado de afectación por la IU expresados por la mujer mediante el cuestionario ICIQ-UI SF, en una muestra de mujeres sexualmente activas que consultaban por IU.

\section{MATERIALES Y MÉTODO}

En el presente análisis se emplearon los datos de las mujeres incluidas en centros de ginecología del estudio UROGINE-QOL, estudio epidemiológico que se realizó en nuestro país para estudiar la relación existente entre el cuestionario ICIQ-UI SF y el KHQ con la CVRS de los pacientes diagnosticados de IU. Concretamente se emplearon los datos de las 1.292 mujeres sexualmente activas del total de 1.735 incluidas en el citado estudio. Se excluyeron 395 mujeres que habían contestado "no procede" en la pregunta sobre "afectación de la vida de pareja (ítem $4 \mathrm{~g})$ del cuestionario KHQ y 48 para las que el ítem se había declarado "missing".

Las pacientes auto-cumplimentaron las versiones españolas, adecuadamente validadas del cuestionario "International Consultation on IncontinenceUrinary Incontinence Short Form" (ICIQ-UI SF) ${ }^{7-9}$ y del "King's Health Questionnaire" (KHQ) ${ }^{10,11}$. Además se recogieron datos generales para definir la población estudiada: edad, peso y talla, para el cálculo del Índice de Masa Corporal-IMC.

El cuestionario ICIQ-UI SF consta de dos grupos de preguntas:
1) 3 preguntas, sobre frecuencia, cantidad y afectación de la calidad de vida, con cuyas respuestas se calcula la puntuación del cuestionario que puede variar entre 0 (continente) y 21 puntos (máximo grado de afectación por la IU);

2) ocho preguntas que se refieren a las situaciones en las que se produce la IU y que permiten orientar el diagnóstico del tipo de IU (de esfuerzo, de urgencia o mixta).

En el presente estudio se excluyeron del estudio aquellos pacientes que respondieron 0 a las preguntas sobre frecuencia y/o cantidad. De acuerdo al ICIQ-UI SF fueron clasificadas como IU de Esfuerzo (IUE) aquellas pacientes con pérdida de orina cuando tose o estornuda y/o con pérdida de orina cuando realiza esfuerzos físicos o ejercicio y que no refieren pérdida de orina antes de llegar al WC; como IU de Urgencia (IUU) las mujeres con pérdida de orina antes de llegar al WC, sin que marcaran como positivas las preguntas sugestivas de IUE; de IU Mixta (IUM) cuando se cumplen ambas condiciones simultáneamente.

El KHQ consta de 21 ítems distribuidos en 9 dimensiones, que se agrupan también en una puntuación global. Además este cuestionario incluye una serie de 11 preguntas en las que se interroga a la paciente sobre los sintomas urinarios que padece y el impacto que causan (leve, moderado o grave). Estas preguntas no forman parte de las puntuaciones de las dimensiones ni de la puntuación global. Se consideró que una mujer padecía IU en el coito cuando marcaba la pregunta correspondiente a "Incontinencia en el acto sexual: escape de orina durante el acto sexual (coito)".

\section{Análisis estadístico}

Los datos fueron analizados con el paquete estadístico SAS versión 9.1 y con SPSS versión 14. Las variables continuas se describieron utilizando la media como medida de tendencia central y la desviación estándar como medida de dispersión. Las variables categóricas se describieron a través de tablas de frecuencia absoluta y relativa. Se compararon la edad y el IMC de las mujeres sin y con IU en el coito, mediante la prueba de la t de Student para muestras independientes. Se estudió la asociación del síntoma de IU en el coito con la de otros síntomas urinarios mediante el análisis de tablas de contingencia y el estadístico Chi Cuadrado. Se analizaron las diferencias en las puntuaciones medias 
de cada una de las 3 preguntas del cuestionario ICIQ-UI SF y la puntuación total, entre las mujeres con y sin IU en el coito, utilizando la prueba de la $\mathrm{t}$ de Student para muestras independientes. Se definió un nivel de significación para todos las pruebas estadísticas de 0,05.

\section{RESULTADOS}

La edad media (D.E.) de las mujeres analizadas $(\mathrm{N}=1.292)$ fue $55,3(11,12)$ años y el IMC medio fue de 26,4 (4,11), teniendo el 43,9\% de ellas, sobrepeso (IMC entre 25 y 30) y siendo el 17,1\% de ellas obesas (IMC>30).

Del total de mujeres analizadas, con sintomas de IU y sexualmente activas, $380(29,4 \%)$ padecían IU en el coito, según las respuestas dadas a la pregunta correspondiente del cuestionario KHQ. Cuando estaba presente, la afectación causada por el síntoma de IU en el coito, declarada por las propias pacientes, fue leve en el $65,7 \%$ de los casos, moderada en el $26,8 \%$ y grave en el $8,4 \%$ de las pacientes.

Las mujeres con IU en el coito tenían similar edad media e IMC que las mujeres que no marcaron ese sintoma al responder el cuestionario KHQ (Tabla 1).

La distribución del total de mujeres analizadas $(\mathrm{N}=1.292)$, respecto de los síntomas de IU de acuerdo a la dimensión de síntomas del cuestionario ICIQ-UI SF, se presenta en la Tabla 2 individualmente cada uno de ellos y agrupados en IU de Esfuerzo, de Urgencia y Mixta.

Las mujeres con síntoma de IU en el coito en relación a las que no presentaron este sintoma, mostraron con mayor frecuencia sintomas de IUE y con menor frecuencia síntomas de IUU, no existiendo diferencias en torno al síntoma de IUM (Tabla 3).

De las mujeres con IUE ( $\mathrm{N}=396), 36,1 \%$ tenian IU en el coito; de las mujeres con IUU ( $\mathrm{N}=313)$, el 20,1\% y de las mujeres con IUM ( $\mathrm{N}=525)$, el 31,8\% ( $\mathrm{p}<0,001)$.

La puntuación total del ICIQ-UI SF fue superior en mujeres con IU en el coito, 14,1 $(3,6)$ que en aquellas $\sin , 12,1(3,8)(p<0,001)$. Las diferencias en la pun-

Tabla 1. Características demográficas de la muestra según refieran o no incontinencia de orina durante la relación sexual coital.

\begin{tabular}{lccc}
\hline Diagnóstico & $\begin{array}{c}\text { Sin IU en el } \\
\text { coito }(\mathbf{N}=\mathbf{9 1 2})\end{array}$ & $\begin{array}{c}\text { Con IU en el } \\
\text { coito }(\mathbf{N}=\mathbf{3 8 0})\end{array}$ & $\mathbf{p}$ \\
\hline Edad media (D.T.) & $55,5(11,6)$ & $54,8(9,9)$ & 0,306 \\
IMC medio (D.T.) & $26,4(4,2)$ & $26,5(3,9)$ & 0,489 \\
\hline
\end{tabular}

T de Student para muestras independientes
Tabla 2. Distribución de la muestra total respecto de los diferentes síntomas urinarios evaluados en el "International Consultation on Incontinence Questionnaire Short Form" (ICIQ-UI SF) y según el tipo de IU basándose en estos sintomas. $(\mathrm{N}=1.292)$

\section{Pérdida de orina según...}

antes de llegar al WC

$838(64,9 \%)$

cuando tose o estornuda

$826(63,9 \%)$

con la realización de esfuerzos físicos

$804(62,2 \%)$

cuando duerme

al acabar de orinar y ya vestido

$121(9,4 \%)$

sin un motivo evidente

$215(16,6 \%)$

$341(26,4 \%)$

de forma continua

$64(5 \%)$

\section{Tipo de IU según los sintomas}

$\begin{array}{ll}\text { IUE aislada* } & 396(30,7 \%) \\ \text { IUU aislada** } & 313(24,2 \%) \\ \text { IU Mixta*** } & 525(40,6 \%)\end{array}$

* IUE : Refiere IU solo ..."cuando tose o estornuda o con la realización de esfuerzos físicos"

** IUU: Refiere IU solo ..."antes de llegar al WC"

*** IU Mixta : Refiere IU ..."cuando tose o estornuda o con la realización de esfuerzos físicos" y "antes de llegar al WC

tuación total del ICIQ-UI SF se debieron fundamentalmente a la diferencia en la dimensión "afectación" más que en "frecuencia” y "cantidad" (Fig. 1).

\section{DISCUSIÓN}

De acuerdo a los resultados presentados, casi una tercera parte de las mujeres que consultan por síntomas de IU y que tienen una relación de pareja y son sexualmente activas, padecen el sintoma de IU durante el coito. De este grupo de mujeres, un tercio califica la afectación causada por la presencia del síntoma de IU en el coito como moderada o grave. Por otro lado la gravedad de la IU medida en base a la puntuación total del cuestionario ICIQ-UI $\mathrm{SF}$, se asocia a la presencia de IU en el coito y al impacto que ésta causa sobre la vida de la paciente.

La limitación principal del trabajo es el método utilizado para seleccionar de entre la muestra las mujeres sexualmente activas: la respuesta al ítem del cuestionario KHQ sobre la afectación de la vida de pareja. Se decidió que la respuesta "no procede" y dejar el ítem en blanco permitía suponer que no eran sexualmente activas. Hay que señalar que se pensó en utilizar los mismos criterios, pero aplicados a la res- 
Tabla 3. Asociación entre la presencia del síntoma de IU en el coito y los síntomas de IU, según el International Consultation on Incontinence Questionnaire Short Form (ICIQ-UI SF) y según el tipo de IU en base a estos síntomas

\begin{tabular}{lccc}
\hline Pérdida de orina según ... & $\begin{array}{c}\text { Sin IU en el coito } \\
\text { (N=912) }\end{array}$ & $\begin{array}{c}\text { Con IU en el coito } \\
\text { (N=380) }\end{array}$ & p \\
\hline antes de llegar al WC & $608(66,7 \%)$ & $230(60,5 \%)$ & 0,021 \\
cuando tose o estornuda & $538(59 \%)$ & $288(75,8 \%)$ & $<0,001$ \\
con la realización de esfuerzos físicos & $521(57,1 \%)$ & $283(74,5 \%)$ & $<0,001$ \\
cuando duerme & $76(8,3 \%)$ & $45(11,8 \%)$ & 0,033 \\
al acabar de orinar y ya vestido & $124(13,6 \%)$ & $91(23,9 \%)$ & $<0,001$ \\
sin un motivo evidente & $225(24,7 \%)$ & $116(30,5 \%)$ & 0,018 \\
de forma continua & $24(2,6 \%)$ & $40(10,5 \%)$ & $<0,001$ \\
\hline Tipo de IU según los síntomas & Sin IU en el coito & Con IU en el coito & p \\
& (N=861) & $143(37,6 \%)$ & $<0,001$ \\
IUE aislada* & $253(27,7 \%)$ & $63(16,6 \%)$ & $<0,001$ \\
IUU aislada** & $250(27,4 \%)$ & $167(43,9 \%)$ & 0,067 \\
IU Mixta*** & $358(39,3 \%)$ & & \\
\hline
\end{tabular}

Chi Cuadrado

* IUE : Refiere IU solo ..."cuando tose o estornuda o con la realización de esfuerzos físicos"

** IUU: Refiere IU solo..." antes de llegar al WC"

*** IU Mixta : Refiere IU..."cuando tose o estornuda o con la realización de esfuerzos fisicos" y "antes de llegar al WC

puesta al ítem del KHQ que pregunta sobre la afectación de la vida sexual; en este caso el número de mujeres no activas sexualmente hubiese resultado ligeramente superior (429 vs 395). Dado el elevado número de coincidencias entre las respuestas a ambos ítems (358 señalaron "no procede" en ambos ítems) y considerando que era menos restrictivo el criterio de "vida de pareja" que el de "vida sexual", se decidió emplear el primer criterio ya señalado.

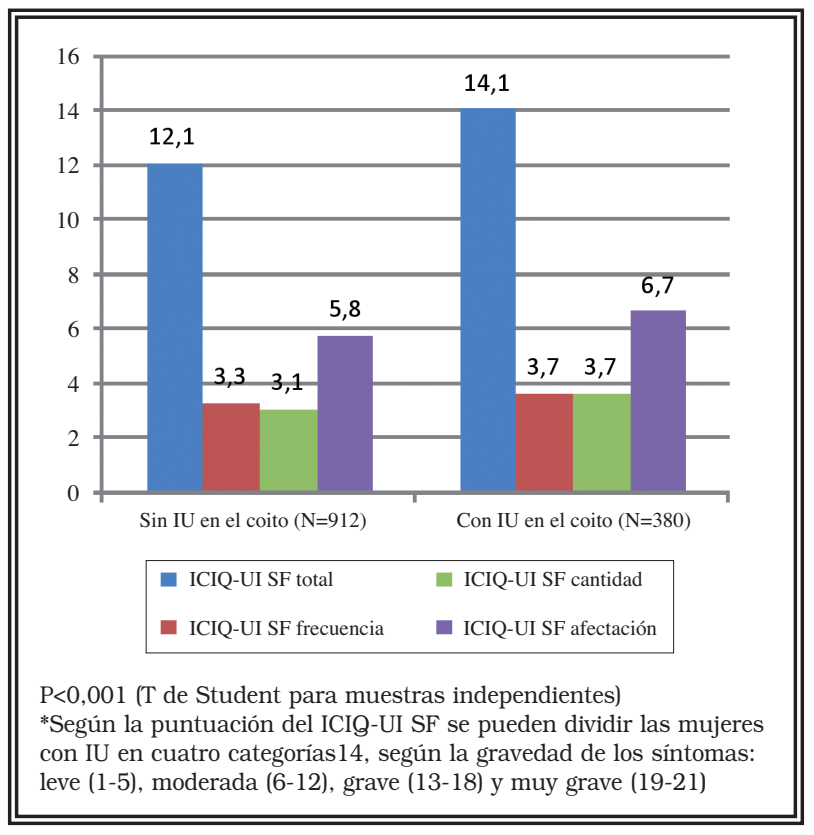

FIGURA 1. Puntuación total en el International Consultation on Incontinence Questionnaire Short Form (ICIQ-UI SF)* según presenten o no IU en el coito.
Casi el 30\% de mujeres estudiadas, con síntomas de IU y sexualmente activas en su relación e pareja, manifestaron tener escapes involuntarios de orina durante la actividad sexual coital, cifra similar al $36,2 \%$ de un estudio previo en otra muestra de mujeres con similares características 1 y en el que se utilizó el mismo criterio (cuestionario KHQ) para su definición. También igual que en el trabajo previo en otra muestra similar de mujeres, no se evidenció asociación de la edad y el IMC con la presencia del síntoma de IU en el coito.

Ambas cifras son algo superiores al 22,8\% de otro estudio que también empleó el cuestionario $\mathrm{KHQ}^{12}$ pero en el que no se seleccionaron a las mujeres sexualmente activas. De hecho si se calcula el porcentaje de mujeres con IU en el coito del presente estudio respecto del total de 1.735 mujeres ( $\sin$ eliminar las mujeres que se asumió no eran sexualmente activas) se obtiene una cifra $(21,9 \%)$ superponible a la del citado estudio. En otros estudios realizados con mujeres diagnosticadas de IU de Esfuerzo sin prolapso genital y sometidas a cirugía, las cifras son sensiblemente superiores. En el estudio de Jha y cols ${ }^{13}$, en un grupo de 72 mujeres con diagnóstico de IUE, tratadas quirúrgicamente con TVT y evaluadas con un cuestionario de síntomas para patología del suelo pélvico "ePAQ", que incluye una dimensión de sexualidad, se encuentra que el 51\% de las mujeres estudiadas refiere IU durante el orgasmo y 33\% durante la penetración. En nuestro estudio, en el grupo de mujeres con IUE, el porcentaje de ellas con IU en la relación sexual fue del 36, 1\%. 
El porcentaje de mujeres con grado de afectación moderada-grave causado por la presencia de este síntoma, fue en el presente trabajo del $34 \%$, algo inferior al del trabajo antes citado $(40,2 \%)^{1}$. Según los resultados presentados sobre la puntuación total del cuestionario ICIQ-UI SF, mayor (peor calidad de vida ) en las mujeres que presentaban IU en el coito que para las que no padecían el síntoma, la IU en el coito no sólo asocia con peor calidad de vida1,3 sino también con mayor gravedad de la IU medida con el cuestionario ICIQ-UI SF ${ }^{14}$.

La mayor frecuencia de sintoma de IU de Esfuerzo y menor de IU de Urgencia en las mujeres con IU en el coito coincide con lo señalado otros estudios ${ }^{5,15}$. En el estudio de Salonia et $\mathrm{al}^{16}$ en el que se evaluó la función sexual con el cuestionario "Female Sexual Function Index (FSI)", se encontró que el $47 \%$ de las mujeres que expresaban disminución del deseo sexual tenían IU de Esfuerzo demostrada con prueba urodinámica, lo que apoyaría la idea de que este tipo de IU se asocia a la IU en el coito más frecuentemente que la IU de urgencia. En el estudio de Jha et $\mathrm{al}^{13}$, recientemente publicado, que analiza el impacto de la corrección quirúrgica de la IUE en la IU durante la función sexual, con la aplicación de una banda libre de tensión (TVT), observa que mejora en la misma proporción la IU en las dos circunstancias descritas, lo cual podría apoyar la hipótesis de que la IU durante la actividad sexual, ya sea la que se produce en la penetración, como la que ocurre con el orgasmos, serían consecuencia de una incompetencia del mecanismo de resistencia uretral. No obstante otros estudios recientes proporcionan interesantes resultados, que indican la existencia de asociación de la presencia de Hiperactividad del Detrusor (HD), demostrada con prueba urodinámica, con la presencia de IU en el momento del orgasmo y no en el momento de la penetración; parece que este grupo de mujeres con $\mathrm{HD}$ e IU en el orgasmo responden peor al tratamiento de la HD con antimuscarínicos ${ }^{6}$.

En resumen, este estudio nos proporciona información sobre la asociación del síntoma IU en el coito con la presencia de los sintomas relacionados con la IUE. También se demuestra que la presencia de IU coital, supone una grado significativamente superior de afectación por la IU , lo cual nos plantea la necesidad de investigar la presencia de este síntoma en mujeres sexualmente activas. Asímismo nos plantea que en estos casos, quizás la indicación de tratamiento quirúrgico de una IUE, debería considerarse aunque los episodios de escape y la intensidad de los mismos no sean muy importantes. Hacen faltan mas estudios prospectivos que evalúen el impacto de los distintos tratamientos de la IU sobre la pérdida de orina relacionada con la actividad sexual.

\section{Agradecimientos}

Este proyecto ha sido financiado por Almirall SA.

\section{REFERENCIAS}

1. Espuña Pons M, Puig Clota, M. Coital urinary incontinence: impact on qualityof life as measured by the King's Health Questionnaire. Int Urogynecol J Pelvic Floor Dysfunct. 2008;19(5):621-625.

2. Espuña Pons M, Puig Clota, M. Sintomas del tracto urinario inferior en la mujer y afectación de la calidad de vida. Resultados de la aplicación del King's Health Questionnaire. Actas Urol Esp. 2006;30:684-691.

3. Oh SJ, Choo MS, Kim HS, Kim JC, Lee JG, Yun JM et al. Generic and disease-specific health-related quality of life in women with coital incontinence: a prospective, multicenter study. Gynecol Obstet Invest. 2008;65(1):62-67

4. Urwitz-Lane R, Ozel B. Sexual function in women with urodynamic stress incontinence, detrusor overactivity and mixed urinary incontinence. Am J Obstet Gynecol. 2006;195(6):1758-1761.

5. Oh SJ, Ku JH, Choo MS, Yun JM, Kim DY, Park WH Health-related quality of life and sexual function in women with stress urinary incontinence and overactive bladder. Int J Urol. 2008:15(1):62-67.

6. Serati M, Salvatore S, Uccella S, Cromi A, Khullar V, Cardozo L et al Urinary incontinence at orgasm: relation to detrusor overactivity and treatmentefficacy. Eur Urol. 2008;54(4):911-915.

7. Avery K, Donovan J, Peters TJ, Shaw C, Gotoh M, Abrams P. A brief and robust measure for evaluating the symptoms and impact of urinary incontinence.Neurourol Urodyn. 2004;23(4):322-330.

8. Espuña Pons M, Puig Clota, M. 2004. Validación de la versión española del ICIQ-SF. Un cuestionario para evaluar la incontinencia urinaria. Med Clin (Barc). 122(8):288-292.

9. Espuña Pons M, Puig Clota M, Rebollo P, Vanrell Díaz JA, Iglesias Guiu $\mathrm{X}$. Evaluación del resultado del tratamiento quirúrgico de la incontinencia urinaria de esfuerzo femenina mediante el cuestionario ICIQ-UI SF. Med Clin (Barc). 2005;124(20):772-774.

10. Kelleher CJ, Cardozo LD, Khullar V, Salvatore S. A new questionnaire to assess the quality of life of urinary incontinent women. BJOG. 1997; 104:1374-1379.

11. Badía Llach X, Castro Díaz D, Conejero Sugrañes J. Validity of the King's Health questionnaire in the assessment of quality of life of patients with urinary incontinence. The King's Group. Med Clin (Barc). 2000; 114(17):647-652.

12. Sand PK, Goldberg RP, Dmochowski RR, Mcllwain M, Dahl NV. The impact of the overactive bladder syndrome on sexual function: a preliminary report from the Multicenter Assessment of Transdermal Therapy in Overactive Bladder with Oxybutynin trial. Am J Obstet Gynecol 2006; 195(6): 1730-5.

13. Jha S, Radley S, Farkas A, Jones G. The impact of TVT on sexual function.Int Urogynecol J Pelvic Floor Dysfunct. 2009;20(2):165-169.

14. Klovning A, Avery K, Sandvik H, Hunskaar S. Comparison of two questionnaires for assessing the severity of urinary incontinence: The ICIQ UI SF versus the incontinence severity index. Neurourol Urodyn. 2009 Feb 12. [Epub ahead of print]

15. Moran PA, Dwyer PL, Ziccone SP. Urinary leakage during coitus in women. J Obstet Gynaecol. 1999;193:286-288.

16. Salonia A, Zanni G, Nappi RE, Briganti A, Dehò F, Fabbri F et al. Sexual dysfunction is common in women with lower urinary tract symptoms and and urinary incontinence: results of a cross-sectional study. Eur Urol. 2004;45:642-648

Correspondencia autor: Dra. Montserrat Espuña Pons ICGON. Hospital Clínic i Provincial

Villarroel, 170 - 08036 Barcelona. Te.1: 932275400

E-mail autor: mespuna@clinic.ub.es

Información artículo: Original - Incontinencia

Trabajo recibido: marzo 2009

Trabajo aceptado: abril 2009 